\title{
Risk of Left Atrial Enlargement in Obese Patients With Obesity-Induced Hypoventilation Syndrome vs Obstructive Sleep Apnea
}

\author{
Yasser Al-Khadra, MD, ${ }^{1}$ Fahed Darmoch, MD, ${ }^{1}$ Mohammad Alkhatib, MD, ${ }^{2}$ Motaz Baibars, MD, ${ }^{3}$ M. Chadi Alraies, $\mathrm{MD}^{4}$ \\ ${ }^{1}$ Department of Internal Medicine, Cleveland Clinic, Cleveland, $\mathrm{OH}^{2}$ Department of Internal Medicine, St. Vincent Charity Medical Center, \\ Cleveland, $\mathrm{OH}^{3}$ Department of Hospital Medicine, Johns Hopkins Medicine, Howard County General Hospital, Columbia, MD ${ }^{4}$ Department of \\ Interventional Cardiology, Wayne State University, Detroit Medical Center, Detroit, MI
}

Background: Obstructive sleep apnea (OSA) is a known risk factor for atrial fibrillation (AF) that is principally driven by left atrial enlargement. The impact of hypoventilation caused by obesity-induced hypoventilation syndrome (OHS) on left atrial diameter has not been examined. We investigated the association between OHS and left atrial diameter in obese patients.

Methods: We performed a retrospective review of 210 consecutive medical records of patients diagnosed as obese (body mass index $[\mathrm{BMI}]>30 \mathrm{~kg} / \mathrm{m}^{2}$ ) and as having OHS and OSA for the period January 2010 through December 2016 at St. Vincent Charity Medical Center in Cleveland, $\mathrm{OH}$. Logistic regression analysis was performed for left atrial diameter $\geq 4 \mathrm{~cm}$ in $2 \mathrm{groups}$ of patients: those with OHS+OSA and those with OSA alone.

Results: A total of 104 obese patients with OHS+OSA and 106 obese patients with OSA alone were identified. Statistically significant differences were found in 6 demographic and baseline characteristics: median BMI, median left atrial diameter, history of type 2 diabetes mellitus, history of stroke, history of coronary artery disease, and history of congestive heart failure. The median left atrial diameter for the OHS+OSA and OSA alone groups was $4.45 \mathrm{~cm}$ and $4.20 \mathrm{~cm}$, respectively $(P=0.014)$. Left ventricular ejection fraction $<50 \%$ was found in $22 \%$ of the patients with OHS+OSA and in $21 \%$ of the patients with OSA alone $(P=0.777)$. Multivariate logistic regression analysis showed that patients in the OHS+OSA group had 2 times higher odds (odds ratio 2.151, 95\% confidence interval 1.016-4.550, $P=0.045$ ) of exhibiting a larger left atrial diameter vs patients in the OSA alone group.

Conclusion: The results of this study indicate that OHS may be an independent risk factor for left atrial enlargement and may possibly contribute to AF development irrespective of left ventricular function.

Keywords: Echocardiography-Doppler, heart atria, obesity hypoventilation syndrome, sleep apnea-obstructive

Address correspondence to M. Chadi Alraies, MD, Department of Cardiology, Wayne State University, Detroit Medical Center, Heart Hospital, 311 Mack Ave., Detroit, MI 48201. Tel: (216) 255-0008. Email: alraies@hotmail.com

\section{INTRODUCTION}

Studies have demonstrated the impact of obstructive sleep apnea (OSA) on cardiovascular morbidity and mortality. Structural and functional remodeling of the left atrium is proportionate to OSA severity and is linked to an increased risk of atrial fibrillation (AF) development. ${ }^{1,2}$ Ninety percent of patients with obesity-induced hypoventilation syndrome (OHS) are also diagnosed with OSA, but patients with OHS have shown a lower overnight peripheral oxygen saturation compared to patients with OSA. ${ }^{3}$ The incidence of cardiovascular diseases such as congestive heart failure (CHF) and atherosclerotic heart disease is higher in OHS patients compared to eucapnic obese and nonobese OSA patients with OSA. ${ }^{4-6}$ Given such differences, whether OHS has an impact similar to OSA on left atrial size remains unclear. This study examined the impact of OHS vs OSA on left atrial diameter. We theorized that persistent hypoxemia caused by OHS increases cardiovascular morbidity and has an effect on left atrial diameter.

\section{METHODS}

We examined the medical records of patients with body mass index $(\mathrm{BMI})>30 \mathrm{~kg} / \mathrm{m}^{2}$ and the diagnoses of $\mathrm{OHS}$ and OSA for the period January 2010 through December 2016 at St. Vincent Charity Medical Center in Cleveland, $\mathrm{OH}$. A total of 210 patients were identified. All the patients with OHS met the diagnostic criteria for this condition. In the OHS+OSA group, $90.4 \%$ of patients had obtainable 
Table 1. Demographic and Baseline Characteristics of Patients With Obesity-Induced Hypoventilation Syndrome (OHA) and Obstructive Sleep Apnea (OSA) vs Patients With OSA Alone

\begin{tabular}{|c|c|c|c|}
\hline Variable & $\begin{array}{c}\text { OHS+OSA } \\
n=104\end{array}$ & $\begin{array}{l}\text { OSA Alone } \\
n=106\end{array}$ & $P$ Value \\
\hline Sex & & & 0.568 \\
\hline Male & $44(42)$ & $49(46)$ & \\
\hline Female & $60(58)$ & $57(54)$ & \\
\hline Age, years, mean $\pm S D$ & $60.25 \pm 12.55$ & $59.36 \pm 11.87$ & 0.598 \\
\hline Body mass index, $\mathrm{kg} / \mathrm{m}^{2}$, median & 47.00 & 43.50 & 0.008 \\
\hline African American & $66(64)$ & $69(65)$ & 0.576 \\
\hline Continuous positive airway pressure compliance & $84(81)$ & $85(80)$ & 0.915 \\
\hline Type 2 diabetes mellitus & $82(79)$ & $67(63)$ & 0.013 \\
\hline Left atrium diameter, $\mathrm{cm}$, median & 4.45 & 4.20 & 0.014 \\
\hline LVEF, \%, median & 55 & 55 & 0.116 \\
\hline LVEF $<50 \%$ & $23(22)$ & $22(21)$ & 0.777 \\
\hline Coronary artery disease & $38(37)$ & $58(55)$ & 0.008 \\
\hline Atrial fibrillation & $23(22)$ & $24(23)$ & 1.000 \\
\hline Congestive heart failure & $77(74)$ & $60(57)$ & 0.008 \\
\hline Stroke & $3(3)$ & $13(12)$ & 0.010 \\
\hline
\end{tabular}

Note: Data are reported as $\mathrm{n}(\%)$ unless otherwise indicated.

LVEF, left ventricular ejection fraction.

documentation of a sleep study. Included patients had a polysomnography study and documented evidence of continuous positive airway pressure (CPAP) treatment.

\section{Echocardiographic Assessment}

Two-dimensional and Doppler echocardiography reports for the studied population were reviewed. Reports were dictated by the cardiologist assigned to the case, and the left atrial diameter was recorded from the cardiologist's assessment. Left atrial diameter $\geq 4 \mathrm{~cm}$ was considered abnormal. Left ventricular ejection fraction (LVEF) was also reported, and an LVEF $<50 \%$ was considered abnormal.

\section{Sleep Disorder Assessment}

OHS was identified in obese patients $\left(\mathrm{BMI}>30 \mathrm{~kg} / \mathrm{m}^{2}\right.$ ) who had sleep-disordered breathing resulting in an awake alveolar hypoventilation $\left(\mathrm{PaCO}_{2}>45 \mathrm{mmHg}\right)$ that could not be attributed to other conditions. OSA was diagnosed by polysomnography in patients with an apnea-hypopnea index $\geq 5$. Apnea was defined as a complete cessation of inspiratory airflow for at least 10 seconds. Hypopnea was defined as a significant reduction ( $>50 \%$ ) of respiratory signals for at least 10 seconds associated with an arousal or oxyhemoglobin desaturation of $3 \%$ or more from baseline.

\section{Statistical Analysis}

Data are expressed as mean \pm standard deviation or as medians, and frequencies are denoted in percentages. Independent 2-sample $t$ tests were used for the comparison of continuous variables measurements, and chi-square test was used for categorical variables. Mann-Whitney $U$ test was used for nonnormally distributed variables. Univariate

Table 2. Univariate and Multivariate Logistic Regression Analyses for the Predictors of Left Atrial Diameter $\geq 4$

\begin{tabular}{|c|c|c|c|c|}
\hline Predictor & $\begin{array}{l}\text { Univariate Odds } \\
\text { Ratio }(95 \% \mathrm{Cl})\end{array}$ & $P$ Value & $\begin{array}{l}\text { Multivariate Odds } \\
\text { Ratio }(95 \% \mathrm{CI})\end{array}$ & $P$ Value \\
\hline Group (OHS+OSA vs OSA alone) & 1.947 (1.040 to 3.647$)$ & 0.037 & 2.151 (1.016 to 4.550$)$ & 0.045 \\
\hline Sex (male vs female) & 2.235 (1.165 to 4.288$)$ & 0.016 & 2.267 (1.112 to 4.619$)$ & 0.024 \\
\hline Age $\geq 75$ years & $1.721(0.556$ to 5.324$)$ & 0.346 & $1.218(0.350$ to 4.233$)$ & 0.757 \\
\hline Body mass index & $1.004(0.978$ to 1.031$)$ & 0.771 & $1.013(0.981$ to 1.046$)$ & 0.440 \\
\hline Type 2 diabetes mellitus & 0.895 (0.443 to 1.704$)$ & 0.663 & $0.583(0.266$ to 1.274$)$ & 0.176 \\
\hline Coronary artery disease & $1.293(0.696$ to 2.402$)$ & 0.416 & $1.743(0.833$ to 3.647$)$ & 0.140 \\
\hline Congestive heart failure & $1.607(0.857$ to 3.013$)$ & 0.139 & $1.368(0.660$ to 2.838$)$ & 0.400 \\
\hline Atrial fibrillation & 3.825 (1.429 to 10.238$)$ & 0.008 & 3.236 (1.143 to 9.163$)$ & 0.027 \\
\hline Left ventricular ejection fraction $<50 \%$ & 1.419 (0.648 to 3.106$)$ & 0.381 & $1.112(0.469$ to 2.635$)$ & 0.809 \\
\hline
\end{tabular}

Cl, confidence interval; OHS, obesity-induced hypoventilation syndrome; OSA, obstructive sleep apnea. 


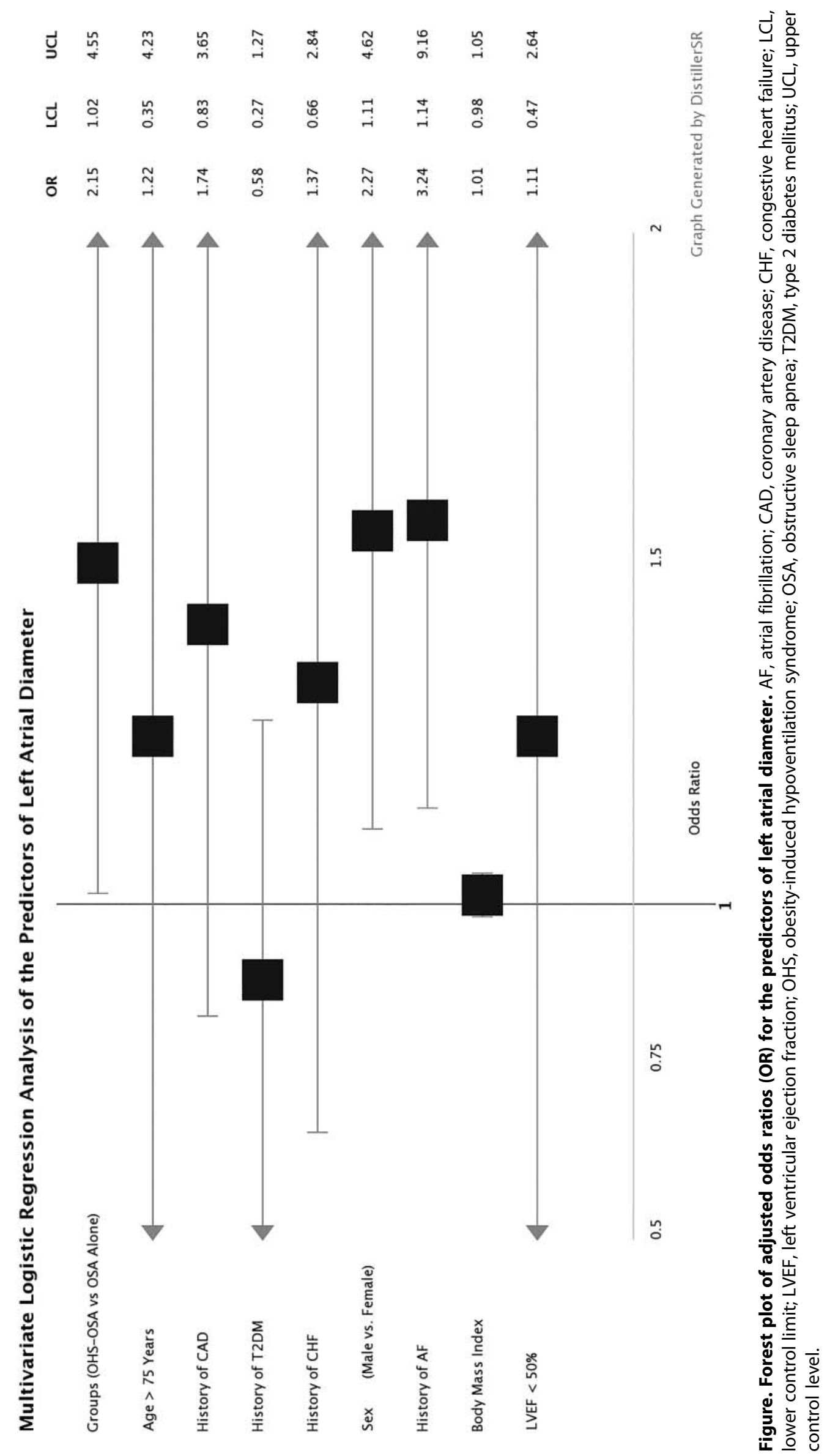


and multivariate logistic regression analyses of left atrial diameter $\geq 4 \mathrm{~cm}$ were used to investigate potential risk factors for left atrial diameter $\geq 4 \mathrm{~cm}$. A Bonferroni correction was applied using all 9 terms in the model, resulting in statistical significance being accepted when $P<0.00454$. $^{7}$ A $P$ value $\leq 0.05$ was considered statistically significant. SPSS v.21 (IBM Corp.) was used for all statistical analyses.

\section{RESULTS}

A total of 104 patients with OHS +OSA and 106 patients with OSA alone were identified. Demographics and baseline characteristics are presented in Table 1. In the OHS+OSA group, $42 \%$ were males compared to $46 \%$ in the OSA alone group $(P=0.568)$. The mean ages (60 vs 59 years, $P=0.598)$ and the proportion of African American patients (64\% vs $65 \%, P=0.576$ ) were comparable in both groups.

Left atrial diameter $(U=4431.5, z=-2.457, P=0.014)$ and BMI $(U=4338, z=-2.667, P=0.008)$ for patients in the OHS+OSA group were statistically higher than for patients in the OSA alone group. Further, type 2 diabetes mellitus and CHF were more prevalent in the OHS+OSA group vs the OSA alone group ( $79 \%$ vs $63 \%, P=0.013$ and $74 \%$ vs $57 \%, P=0.008$, respectively). On the other hand, the OHS + OSA alone group had a lower prevalence of coronary artery disease and stroke compared with the OSA alone group ( $37 \%$ vs $55 \%, P=0.008$ and $3 \%$ vs $12 \%, P=0.010$, respectively). However, the median LVEF in the OHS+OSA and OSA alone groups was not statistically different $(U=4419.5, z=-1.574, P=0.116)$.

Multivariate logistic regression analysis investigated potential risk factors for left atrial diameter $\geq 4 \mathrm{~cm}$ : group (OHS+OSA vs OSA alone), age $\geq 75$ years, sex, BMI, type 2 diabetes mellitus, coronary artery disease, CHF, AF, and LVEF $<50 \%$. Of these 9 predictor variables, 3 were statistically significant: group, sex, and AF (Table 2). Patients in the OHS+OSA group had 2 times higher odds (odds ratio $2.151,95 \%$ confidence interval $1.016-4.550, P=0.045$ ) of exhibiting a larger left atrial diameter vs patients in the OSA alone group. Being male and having a history of AF were also associated with an increased likelihood of exhibiting a larger left atrial diameter (Figure) in the OHS+OSA vs OSA alone groups.

\section{DISCUSSION}

Our findings suggest a significant association between a larger left atrial diameter in patients with $\mathrm{OHS}+\mathrm{OSA}$ compared to patients with OSA alone, and the OHS association with a larger left atrial diameter remained significant after adjusting for age, BMl, sex, type 2 diabetes mellitus, coronary artery disease, CHF, AF, and LVEF. OHS appears to be an independent risk factor for left atrial enlargement in obese patients. In our cohort, patients with OHS+OSA were found to have 2-fold increased odds for left atrial diameter $\geq 4 \mathrm{~cm}$ vs patients with OSA alone. OSA is associated with cardiovascular risk and left atrial enlargement as a result of repetitive nocturnal hypoxemia. ${ }^{2,8-10}$ Despite the close relationship between OSA and OHS, patients with OHS have a notably lower overnight hypoxemia and therefore higher cardiovascular morbidity compared to OSA patients. To our knowledge, no study has examined the effect of OHS vs OSA on left atrial diameter given the difference in hypoxia degree.
Neither the univariate nor the multivariate analysis showed a statistically significant LVEF effect on the results. However, males had a 2 -fold higher risk of having a larger left atrial diameter in both the univariate and the multivariate analysis. Similarly, patients with a history of AF had a 3-fold increase in risk of left atrial enlargement compared to non-AF patients, a finding in keeping with the Framingham Heart Study that demonstrated a $39 \%$ risk increase of AF development with each 5-mm left atrial diameter enlargement. ${ }^{11,12}$ Although the cardiovascular risk associated with OHS has been studied, left atrial remodeling as a result of $\mathrm{OHS}$ has not yet been explored.

OHS and OSA are modifiable risk factors, and noninvasive ventilatory support treatment has been shown to lower AF recurrence after catheter ablation therapy in patients with OSA. ${ }^{13}$ Therefore, directing further attention to the management and treatment of OHS in patients with cardiovascular risk could potentially benefit this patient group by reducing cardiovascular morbidity and mortality. Although the Sleep Apnea Cardiovascular Endpoints (SAVE) trial showed no benefit of CPAP treatment in improving cardiovascular mortality, the trial excluded patients with comorbidities, severe nocturnal hypoxia, or prior CPAP treatment. ${ }^{14}$ The effect of OHS treatment and CPAP compliance on left atrial size needs further evaluation.

Our study has several limitations as it is a retrospective study with a relatively small sample size. Although both the OSA and OHS patients had a polysomnography and a record of CPAP treatment, information regarding the diagnosis duration, OSA severity, and duration of CPAP compliance were missing in each group.

\section{CONCLUSION}

We identified $\mathrm{OHS}$ as a potential independent risk factor for left atrial enlargement. Given that OHS is a modifiable risk factor, proper screening and management of OHS may decrease preventable cardiovascular morbidity-related atrial remodeling. The effect on cardiovascular morbidity and mortality of noninvasive ventilatory measures in patients with $\mathrm{OHS}$ is uncertain. Furthermore, the association between left atrial diameter and AF needs to be validated in further studies.

\section{ACKNOWLEDGMENTS}

The authors have no financial or proprietary interest in the subject matter of this article.

\section{REFERENCES}

1. Kim SM, Cho KI, Kwon JH, Lee HG, Kim TI. Impact of obstructive sleep apnea on left atrial functional and structural remodeling beyond obesity. J Cardiol. 2012 Dec;60(6):475-483. doi: 10 .1016/j.jjcc.2012.07.007.

2. Oliveira W, Campos O, Bezerra Lira-Filho E, et al. Left atrial volume and function in patients with obstructive sleep apnea assessed by real-time three-dimensional echocardiography. J Am Soc Echocardiogr. 2008 Dec;21(12):1355-1361. doi: 10.1016/ j.echo.2008.09.007.

3. Pihtili A, Bingöl Z, Kiyan E. The predictors of obesity hypoventilation syndrome in obstructive sleep apnea. Balkan Med J. 2017 Jan;34(1):41-46. doi: 10.4274/ balkanmedj.2015.1797. 
4. Kessler R, Chaouat A, Schinkewitch $P$, et al. The obesityhypoventilation syndrome revisited: a prospective study of 34 consecutive cases. Chest. 2001 Aug;120(2):369-376.

5. Akashiba T, Akahoshi T, Kawahara S, et al. Clinical characteristics of obesity-hypoventilation syndrome in Japan: a multi-center study. Intern Med. 2006;45(20): 1121-1125.

6. Kessler R, Chaouat A, Weitzenblum E, et al. Pulmonary hypertension in the obstructive sleep apnoea syndrome: prevalence, causes and therapeutic consequences. Eur Respir J. 1996 Apr;9(4):787-794.

7. Tabachnick BG, Fidell LS. Using Multivariate Statistics. 6th ed. Essex, UK: Pearson; 2014.

8. Benjamin EJ, D'Agostino RB, Belanger AJ, Wolf PA, Levy D. Left atrial size and the risk of stroke and death. The Framingham Heart Study. Circulation. 1995 Aug 15;92(4): 835-841.

9. Phillips B. Sleep-disordered breathing and cardiovascular disease. Sleep Med Rev. 2005 Apr;9(2):131-140.
10. Marin JM, Carriz SJ, Vicente E, Agusti AG. Long-term cardiovascular outcomes in men with obstructive sleep apnoea-hypopnoea with or without treatment with continuous positive airway pressure: an observational study. Lancet. 2005 Mar 19-25;365(9464):1046-1053.

11. Patel DA, Lavie CJ, Milani RV, Shah S, Gilliland Y. Clinical implications of left atrial enlargement: a review. Ochsner J. 2009 Winter;9(4):191-196.

12. Vaziri SM, Larson MG, Benjamin EJ, Levy D. Echocardiographic predictors of nonrheumatic atrial fibrillation. The Framingham Heart Study. Circulation. 1994 Feb;89(2):724-730.

13. Fein AS, Shvilkin A, Shah D, et al. Treatment of obstructive sleep apnea reduces the risk of atrial fibrillation recurrence after catheter ablation. J Am Coll Cardiol. 2013 Jul 23;62(4):300305. doi: 10.1016/j.jacc.2013.03.052.

14. McEvoy RD, Antic NA, Heeley E, et al; SAVE Investigators and Coordinators. CPAP for prevention of cardiovascular events in obstructive sleep apnea. N Engl J Med. 2016 Sep 8;375(10):919931. doi: 10.1056/NEJMoa1606599.

This article meets the Accreditation Council for Graduate Medical Education and the American Board of Medical Specialties Maintenance of Certification competencies for Patient Care and Medical Knowledge. 